\title{
On Modelling of Generalized Peterson's Syllogisms with More Premises
}

\author{
Karel Fiala $^{a}$ and Veronika Červeňová ${ }^{b}$ and Petra Murinovác \\ ${ }^{a}$ Department of Mathematics, Faculty of Science, University of Ostrava, \\ 30. dubna 22, 70103 Ostrava 1, Czech Republic, karel.fiala@osu.cz \\ ${ }^{b}$ Department of Mathematics, Faculty of Science, University of Ostrava, \\ 30. dubna 22, 70103 Ostrava 1, Czech Republic, veronika. cervenova@osu.cz \\ ${ }^{c}$ Institute for Research and Applications of Fuzzy Modelling, University of Ostrava, \\ 30. dubna 22, 70103 Ostrava 1, Czech Republic, petra.murinova@osu.cz
}

\begin{abstract}
This article aims to continue the analysis of generalized Peterson's syllogisms with more premises. Namely, we use the theory of intermediate quantifiers, which provides the mathematical interpretation of natural language expressions indicating the amount or quantity of objects, such as "Almost all", "Several" etc. In the second part of this paper, we propose an algorithm for the computation of truth degrees of expressions containing such quantifiers and verify the validity/invalidity of several syllogisms.
\end{abstract}

Keywords: Generalized Intermediate Quantifiers, Generalized Syllogisms, Validity/Invalidity of Syllogisms.

\section{Introduction}

Quantified statements are an essential part of our language. We use statements such as "most people", "some children" daily to describe quantities of objects or people. From classical mathematics, we know the universal quantifier $\forall$ (for all) and the existential quantifier $\exists$ (there exists). We modify their meaning using evaluative linguistic expressions to arrive at new quantifiers (Almost all, Many, Several, etc.) Since their meaning lies "between" the classical quantifiers, we call them intermediate.

In real life, we naturally deduce new information or draw a conclusion from given premises. We call this reasoning a syllogism. Classical logical syllogisms, as well as graded syllogisms, were analyzed mainly from the point of view of their semantic interpretation by several authors $[13,14,2,16]$. This article aims to build on the results [4] achieved regarding the interpretation of generalized Peterson's syllogisms which contain expressions of natural language such as "Almost all, A few, Several", etc. The first idea is to extend Peterson's basic quantifiers [15] by new forms and to verify the validity of corresponding syllogisms with more premises. Following these generalized syllogisms, we will show how these syllogisms can be transformed into well known generalized Peterson's syllogisms which were studied in [4, 3] from a syntactic and semantic point of view. The second part of this paper is dedicated to proposing an algorithm for the evaluation of intermediate quantifiers and implementing it in evaluating syllogisms with more premises.

The novelty of this approach is to use the proposed algorithm and to verify the validity/invalidity of generalized syllogisms. As a result, we obtain from the validity of the following sentences:

- All species are mammals.

- Almost all species of whales are threatened with extinction.

the truth degree of the following conclusion:

- Some species of whales that are threatened with extinction are mammals.

A very important application of intermediate quantifiers and their syllogisms can be found in fuzzy association analysis. Using this approach, we can derive new information from data.

\section{Definition of Intermediate Quantifiers}

In this paper, we will deal with the model of intermediate quantifiers which was introduced by Novák in [9] within Łukasiewicz fuzzy type theory (Ł-FTT). We omit although precise definitions, which can be found in detail elsewhere $[9,7,4,5,8,10]$. The basic syntactic objects of Ł-FTT are classical, namely the concepts 
of type and formula (see [1]). The atomic types are $\varepsilon$ (elements) and $o$ (truth values). General types are defined as follows: if $\alpha, \beta$ are types then $(\beta \alpha)$ is a type. We denote types by Greek letters and the set of all types by Types. The set of all formula of a type $\alpha$ is denoted by Types $\alpha$.

The model deals with intermediate quantifiers of type $\langle 1,1\rangle$ which were expressed by the abovementioned examples.

For modelling of intermediate quantifiers, we use evaluative linguistic expressions, fuzzy measure and a special operation "cut of a fuzzy set".

The theory of intermediate quantifiers is based on the theory of evaluative linguistic expressions that are expressions of natural language such as small, medium, big, very short, more or less deep, quite roughly strong, extremely high, etc. There is a formal theory of them presented in detail in [8] and less formally including formulas for the direct computation in [12].

A very important role is the one of fuzzy measure which is formally defined as follows:

Definition 1. Let $R \in$ Form $_{o(o \alpha)(o \alpha)}$ be a formula where $\alpha \in$ Types is an arbitrary type.

A formula $\mu \in$ Form $_{o(o \alpha)(o \alpha)}$ defined by

$$
\mu_{o(o \alpha)(o \alpha)} \equiv z_{o \alpha} x_{o \alpha}\left(R z_{o \alpha}\right) x_{o \alpha}
$$

represents a measure on fuzzy sets in the universe of type $\alpha \in$ Types if it has the following properties:

(M1) $\boldsymbol{\Delta}\left(x_{o \alpha} \subseteq z_{o \alpha}\right) \boldsymbol{\&} \boldsymbol{\Delta}\left(y_{o \alpha} \subseteq z_{o \alpha}\right) \boldsymbol{\&} \boldsymbol{\Delta}\left(x_{o \alpha} \subseteq y_{o \alpha}\right) \Rightarrow$ $\left(\left(\mu z_{o \alpha}\right) x_{o \alpha} \Rightarrow\left(\mu z_{o \alpha}\right) y_{o \alpha}\right)$,

(M2) $\boldsymbol{\Delta}\left(x_{o \alpha} \subseteq z_{o \alpha}\right) \Rightarrow\left(\left(\mu z_{o \alpha}\right)\left(z_{o \alpha} \backslash x_{o \alpha}\right) \equiv\right.$ $\left.\neg\left(\mu z_{o \alpha}\right) x_{o \alpha}\right)$,

(M3) $\boldsymbol{\Delta}\left(x_{o \alpha} \subseteq y_{o \alpha}\right) \boldsymbol{\&} \boldsymbol{\Delta}\left(x_{o \alpha} \subseteq z_{o \alpha}\right) \boldsymbol{\&} \boldsymbol{\Delta}\left(y_{o \alpha} \subseteq z_{o \alpha}\right) \Rightarrow$ $\left(\left(\mu z_{o \alpha}\right) x_{o \alpha} \Rightarrow\left(\mu y_{o \alpha}\right) x_{o \alpha}\right)$

where $x_{o \alpha}, y_{o \alpha}, z_{o \alpha}$ are variables representing fuzzy sets.

The following formula characterizes measurable fuzzy sets of a given type $\alpha$ :

$$
\begin{array}{r}
\mathbf{M}_{o(o \alpha)} \equiv z_{o \alpha} \cdot \neg \boldsymbol{\Delta}\left(z_{o \alpha} \equiv \emptyset_{o \alpha}\right) \boldsymbol{\&} \boldsymbol{\Delta}\left(\mu z_{o \alpha}\right) z_{o \alpha} \boldsymbol{\&} \\
\left(\forall x_{o \alpha}\right)\left(\forall y_{o \alpha}\right) \boldsymbol{\Delta}((M 1) \boldsymbol{\&}(M 3)) \boldsymbol{\&}\left(\forall x_{o \alpha}\right) \boldsymbol{\Delta}(M 2)
\end{array}
$$

where (M1)-(M3) are the axioms from (i).

Finally, we define a special operation "cut of a fuzzy set" for given fuzzy sets $y, z \in$ Form $_{o \alpha}$ :

$$
y \mid z \equiv \lambda x_{\alpha} \cdot z x \& \Delta\left(\Upsilon^{1}(z x) \Rightarrow(y x \equiv z x)\right) .
$$

\footnotetext{
${ }^{1}$ The formal definition is $\Upsilon_{o o} \equiv \lambda z_{o} \cdot \neg \boldsymbol{\Delta}\left(\neg z_{o}\right)$.
}

Lemma 1. [6] Let $\mathscr{M}$ be a model and $p$ an assignment such that $B=\mathscr{M}_{p}(y) \Subset M_{\alpha}, Z=\mathscr{M}_{p}(z) \Subset M_{\alpha}$. Then for any $m \in M_{\alpha}$

$\mathscr{M}_{p}(y \mid z)(m)=(B \mid Z)(m)= \begin{cases}B(m), & \text { if } B(m)=Z(m), \\ 0 & \text { otherwise. }\end{cases}$

By this operation, we can create subsets of the fuzzy set $B$. We may notice that the operation either selects an element from $B$ exactly in its degree of membership or does not select it at all. Now we can present the mathematical definition of intermediate quantifiers:

Definition 2. Let $E v$ be a formula representing an evaluative expression, $x$ be variables and $A, B, z$ be formulas. Then either of the formulas represents the quantifier "Quantifier B's are A”.

$\left(Q_{E v}^{\forall} x\right)(B, A) \equiv(\exists z)[(\forall x)((B \mid z) x \Rightarrow A x) \wedge E v((\mu B)(B \mid z))]$,

$\left(Q_{E v}^{\exists} x\right)(B, A) \equiv(\exists z)[(\exists x)((B \mid z) x \wedge A x) \wedge E v((\mu B)(B \mid z))]$.

If we replace $E v$ in (3), (4) by a specific evaluative linguistic expression, we get the definition of a particular intermediate quantifier. Quantifiers A,P,T,K,F,S,I are positive and quantifiers $\mathbf{E}, \mathbf{B}, \mathbf{D}, \mathbf{G}, \mathbf{V}, \mathbf{Z}, \mathbf{O}$ are negative.

Definition 3. [6, 11]

$$
\begin{aligned}
\text { A: All B are } A & :=(\forall x)(B x \Rightarrow A x), \\
\text { E: No B are } A & :=(\forall x)(B x \Rightarrow \neg A x), \\
\text { P: Almost all B are } A & :=\left(Q_{B i E x}^{\forall} x\right)(B, A),
\end{aligned}
$$

B: Almost all $B$ are not $A:=\left(Q_{B i E x}^{\forall} x\right)(B, \neg A)$,

$$
\text { T: } \text { Most } B \text { are } A:=\left(Q_{B i v e}^{\forall} x\right)(B, A),
$$

D: Most $B$ are not $A:=\left(Q_{B i V e}^{\forall} x\right)(B, \neg A)$,

$$
\text { K: Many } B \text { are } A:=\left(Q_{\neg S m \overline{\mathbf{v}}}^{\forall} x\right)(B, A),
$$

G: Many $B$ are not $A:=\left(Q_{\neg S m}^{\forall} x\right)(B, \neg A)$, $\boldsymbol{F}: A$ few $B$ are $A:=\left(Q_{S m S i}^{\forall} x\right)(B, A)$,

$V: A$ few $B$ are not $A:=\left(Q_{S m S i}^{\forall} x\right)(B, \neg A)$,

S: Several B are $A:=\left(Q_{S m V e}^{\forall} x\right)(B, A)$,

$Z$ : Several $B$ are not $A:=\left(Q_{S m V e}^{\forall} x\right)(B, \neg A)$,

I: Some $B$ are $A:=(\exists x)(B x \wedge A x)$

$\boldsymbol{O}$ : Some $B$ are not $A:=(\exists x)(B x \wedge \neg A x)$.

By *A, *E, *P, *B, *T, *D, *K, *G, *F, *V, *S, *Z, *I, $* \boldsymbol{O}$ we denote intermediate quantifiers with presupposition. For the precise definition see [6].

In the following theorem, we will show the monotonicity of positive and negative intermediate quantifiers. 
Theorem 1. [11] Let $\boldsymbol{A}, \ldots, \boldsymbol{O}$ are intermediate quantifiers. Then the following set of implications is provable in $T^{I Q}$ :

$$
\begin{array}{ll}
\text { 1. } & T^{I Q} \vdash \boldsymbol{A} \Rightarrow \boldsymbol{P}, T^{I Q} \vdash \boldsymbol{P} \Rightarrow \boldsymbol{T}, T^{I Q} \vdash \boldsymbol{T} \Rightarrow \boldsymbol{K}, \\
& T^{I Q} \vdash \boldsymbol{K} \Rightarrow \boldsymbol{F}, T^{I Q} \vdash \boldsymbol{F} \Rightarrow \boldsymbol{S}, T^{I Q} \vdash \boldsymbol{S} \Rightarrow \boldsymbol{I} . \\
\text { 2. } & T^{I Q} \vdash \boldsymbol{E} \Rightarrow \boldsymbol{B}, T^{I Q} \vdash \boldsymbol{B} \Rightarrow \boldsymbol{D}, T^{I Q} \vdash \boldsymbol{D} \Rightarrow \boldsymbol{G}, \\
& T^{I Q} \vdash \boldsymbol{G} \Rightarrow \boldsymbol{V}, T^{I Q} \vdash \boldsymbol{V} \Rightarrow \boldsymbol{Z}, T^{I Q} \vdash \boldsymbol{Z} \Rightarrow \boldsymbol{O} .
\end{array}
$$

\section{Generalized Syllogisms with Three Premises}

By the classical definition, a categorical syllogism is a special kind of logical argument in which we infer the conclusion from two premises. For inference of the conclusion is used an assumption of a middle formula. Generalized syllogisms are obtained by replacing the classical quantifier with the intermediate quantifier. For syllogisms with two premises, there are four corresponding figures.

In this paper, we will extend this theory by adding another premise. We have three premises $\left(P_{1}, P_{2}, P_{3}\right)$ from which we want to derive the conclusion $(C)$. For this, we need to assume two middle formulas $\left(M_{1}, M_{2}\right)$ and we get eight corresponding figures. We can express a syllogism with three premises by a foursome of formulas $\left\langle P_{1}, P_{2}, P_{3}, C\right\rangle$.

Definition 4. Syllogism $\left\langle P_{1}, P_{2}, P_{3}, C\right\rangle$ is strongly valid in $T^{I Q}$ if $T^{I Q} \vdash\left(P_{1} \& P_{2} \& P_{3}\right) \Rightarrow C$ or equivalently if $T^{I Q} \vdash P_{1} \Rightarrow\left(P_{2} \Rightarrow\left(P_{3} \Rightarrow C\right)\right)$.

Definition 5. Let $Q_{1}, Q_{2}, Q_{3}, Q_{4}$ be intermediate quantifiers. Let $M_{1}, M_{2}$ be middle formulas, let $S$ be subject and let $P$ be predicate. $M_{1}, M_{2}, P, S \in$ Form $_{o \alpha}$.

Below we introduce the list of figures with two middle's formula.

\section{Figure-I}

$P_{1}: Q_{1} M_{1}$ is $P$ $P_{2}: Q_{2} M_{2}$ is $M_{1}$ \begin{tabular}{l}
$P_{3}: Q_{3} S$ is $M_{2}$ \\
\hline$C: Q_{4} S$ is $P$
\end{tabular}

Figure-IV

$Q_{1} P$ is $M_{1}$ $Q_{2} M_{2}$ is $M_{1}$

$\frac{Q_{3} S \text { is } M_{2}}{Q_{4} S \text { is } P}$

Figure-VII

$Q_{1} P$ is $M_{1}$

$Q_{2} M_{1}$ is $M_{2}$

$Q_{3} M_{2}$ is $S$
$Q_{4} S$ is $P$

\section{Figure-II}

$Q_{1} M_{1}$ is $P$

$Q_{2} M_{2}$ is $M_{1}$

$Q_{3} M_{2}$ is $S$
$Q_{4} S$ is $P$

\section{Figure-V}

$Q_{1} M_{1}$ is $P$

$Q_{2} M_{1}$ is $M_{2}$

$\frac{Q_{3} M_{2} \text { is } S}{Q_{4} S \text { is } P}$
To write generalized syllogisms on Figure-I, we use the abbreviation $Q_{1} Q_{2} Q_{3} Q_{4}$-I. We use this abbreviation analogously for other figures.

\subsection{Figure II}

In the following subsections, we will deal with FigureII. In section 3.1.1, we will construct formal mathematical proofs and then we will show how we can use the monotonicity of quantifiers. In section 3.1.2, we will show another approach on how to show strong validity of syllogisms with three premises by converting them to two syllogisms with two premises.

\subsubsection{Syntactic Proofs}

Firstly we will syntactically prove some syllogisms on this figure.

Theorem 2. The syllogisms AAII, APKI, ATTI, AKPI, AIAI are strongly valid in $T^{I Q}$.

$$
\begin{array}{ll} 
& (\forall x)\left(M_{1} x \Rightarrow P x\right) \\
\text { Proof. AAII-II: } & (\forall x)\left(M_{2} x \Rightarrow M_{1} x\right) \\
& (\exists x)\left(M_{2} x \wedge S x\right) \\
& (\exists x)(S x \wedge P x)
\end{array}
$$

The following formulas are strongly valid in $T^{I Q}$ : $T^{I Q} \vdash\left(\left(M_{1} x \Rightarrow P x\right) \&\left(M_{2} x \Rightarrow M_{1} x\right)\right) \Rightarrow\left(M_{2} x \Rightarrow P x\right)$ and $T^{I Q} \vdash\left(M_{2} x \Rightarrow P x\right) \Rightarrow\left(\left(M_{2} x \wedge S x\right) \Rightarrow(S x \wedge P x)\right)$. Then by transitivity and by applying the of rule MP, we obtain

$$
\begin{aligned}
T^{I Q} \vdash\left(\left(M_{1} x \Rightarrow P x\right)\right. & \left.\&\left(M_{2} x \Rightarrow M_{1} x\right)\right) \Rightarrow \\
& \Rightarrow\left(\left(M_{2} x \wedge S x\right) \Rightarrow(S x \wedge P x)\right) .
\end{aligned}
$$

By adjunction we obtain

$$
\begin{aligned}
& T^{I Q} \vdash\left(M_{1} x \Rightarrow P x\right) \Rightarrow \\
& \Rightarrow\left(\left(M_{2} x \Rightarrow M_{1} x\right) \Rightarrow\left(\left(M_{2} x \wedge S x\right) \Rightarrow(S x \wedge P x)\right)\right) .
\end{aligned}
$$

By the generalization rule $(\forall x)$ and by properties of quantifiers we obtain

$$
\begin{array}{r}
T^{I Q} \vdash(\forall x)\left(M_{1} x \Rightarrow P x\right) \Rightarrow\left((\forall x)\left(M_{2} x \Rightarrow M_{1} x\right) \Rightarrow\right. \\
\left.\Rightarrow\left((\exists x)\left(M_{2} x \wedge S x\right) \Rightarrow(\exists x)(S x \wedge P x)\right)\right) .
\end{array}
$$

Finally, we use adjunction and we obtain

$$
\begin{gathered}
T^{I Q} \vdash\left((\forall x)\left(M_{1} x \Rightarrow P x\right) \&(\forall x)\left(M_{2} x \Rightarrow M_{1} x\right) \&\right. \\
\left.\&(\exists x)\left(M_{2} x \wedge S x\right)\right) \Rightarrow(\exists x)(S x \wedge P x) .
\end{gathered}
$$

$Q_{2} M_{1}$ is $M_{2}$

$Q_{3} S$ is $M_{2}$
$Q_{4} S$ is $P$

Figure-VIII

$Q_{1} P$ is $M_{1}$

$Q_{2} M_{1}$ is $M_{2}$

$Q_{3} S$ is $M_{2}$
$Q_{4} S$ is $P$ $(\forall x)\left(M_{1} x \Rightarrow P x\right)$

Proof. AIAI-II. $\quad(\exists x)\left(M_{2} x \wedge M_{1} x\right)$

$\frac{(\forall x)\left(M_{2} x \Rightarrow S x\right)}{(\exists x)(S x \wedge P x)}$ 
The following formulas are strongly valid in $T^{I Q}$ : $T^{I Q} \vdash\left(\left(M_{1} x \Rightarrow P x\right) \&\left(M_{2} x \wedge M_{1} x\right)\right) \Rightarrow\left(M_{2} x \wedge P x\right)$ and $T^{I Q} \vdash\left(M_{2} x \wedge P x\right) \Rightarrow\left(\left(M_{2} x \Rightarrow S x\right) \Rightarrow(S x \wedge P x)\right)$. By transitivity and by MP we obtain

$$
\begin{aligned}
& T^{I Q} \vdash\left(\left(M_{1} x \Rightarrow P x\right) \&\left(M_{2} x \wedge M_{1} x\right)\right) \Rightarrow \\
& \quad \Rightarrow\left(\left(M_{2} x \Rightarrow S x\right) \Rightarrow(S x \wedge P x)\right) .
\end{aligned}
$$

By adjunction we obtain

$$
\begin{aligned}
& T^{I Q} \vdash\left(M_{1} x \Rightarrow P x\right) \Rightarrow \\
& \Rightarrow\left(\left(M_{2} x \Rightarrow S x\right) \Rightarrow\left(\left(M_{2} x \wedge M_{1} x\right) \Rightarrow(S x \wedge P x)\right)\right) .
\end{aligned}
$$

By the generalization rule $(\forall x)$ and by quantifiers properties we obtain

$$
\begin{gathered}
T^{I Q} \vdash(\forall x)\left(M_{1} x \Rightarrow P x\right) \Rightarrow\left((\forall x)\left(M_{2} x \Rightarrow S x\right) \Rightarrow\right. \\
\left.\Rightarrow\left((\exists x)\left(M_{2} x \wedge M_{1} x\right) \Rightarrow(\exists x)(S x \wedge P x)\right)\right) .
\end{gathered}
$$

Finally we use adjunction and we obtain

$$
\begin{gathered}
T^{I Q} \vdash\left((\forall x)\left(M_{1} x \Rightarrow P x\right) \&(\exists x)\left(M_{2} x \wedge M_{1} x\right) \&\right. \\
\left.\&(\forall x)\left(M_{2} x \Rightarrow S x\right)\right) \Rightarrow(\exists x)(S x \wedge P x) .
\end{gathered}
$$

We can see that syllogisms AIAI-II and AAII-II contain only classical quantifiers.

$$
\begin{aligned}
& \text { Proof. APKI-II: } \\
& (\forall x)\left(M_{1} x \Rightarrow P x\right) \\
& (\exists z)\left[(\forall x)\left(\left(M_{2} \mid z\right) x \Rightarrow M_{1} x\right) \wedge \operatorname{BiEx}\left(\left(\mu M_{2}\right)\left(M_{2} \mid z\right)\right)\right] \\
& \left(\exists z^{\prime}\right)\left[(\forall x)\left(\left(M_{2} \mid z^{\prime}\right) x \Rightarrow S x\right) \wedge \neg \operatorname{Sm} \overline{\mathbf{v}}\left(\left(\mu M_{2}\right)\left(M_{2} \mid z^{\prime}\right)\right)\right] \\
& \hline(\exists x)(S x \wedge P x)
\end{aligned}
$$

\section{Proof. APKI-II:}

Let $E v:=\operatorname{BiEx}\left(\left(\mu M_{2}\right)\left(M_{2} \mid z\right)\right)$ and $E v^{\prime}:=$ $\neg \operatorname{Sm} \overline{\mathbf{v}}\left(\left(\mu M_{2}\right)\left(M_{2} \mid z^{\prime}\right)\right)$ The following formula is strongly valid: $T^{I Q} \vdash\left(M_{1} x \Rightarrow P x\right) \Rightarrow\left(\left(\left(M_{2} \mid z\right) x \Rightarrow\right.\right.$ $\left.\left.M_{1} x\right) \Rightarrow\left(\left(M_{2} \mid z\right) x \Rightarrow P x\right)\right)$. By the generalization rule $(\forall x)$ and by properties of quantifiers we obtain $T^{I Q} \vdash(\forall x)\left(M_{1} \Rightarrow P x\right) \Rightarrow\left((\forall x)\left(\left(M_{2} \mid z\right) x \Rightarrow M_{1} x\right) \Rightarrow\right.$ $\left.(\forall x)\left(\left(M_{2} \mid z\right) x \Rightarrow P x\right)\right)$. By Ł-FTT properties we obtain

$$
\begin{aligned}
& T^{I Q} \vdash(\forall x)\left(M_{1} \Rightarrow P x\right) \Rightarrow\left(\left[( \forall x ) \left(\left(M_{2} \mid z\right) x \Rightarrow\right.\right.\right. \\
& \left.\left.\left.\quad \Rightarrow M_{1} x\right) \wedge E v\right] \Rightarrow\left[(\forall x)\left(\left(M_{2} \mid z\right) x \Rightarrow P x\right) \wedge E v\right]\right) .
\end{aligned}
$$

Finally, by adjunction we obtain

$$
\begin{aligned}
& T^{I Q} \vdash\left((\forall x)\left(M_{1} \Rightarrow P x\right) \&\right. \\
& \left.\&\left[(\forall x)\left(\left(M_{2} \mid z\right) x \Rightarrow M_{1} x\right) \wedge E v\right]\right) \Rightarrow \\
& \quad \Rightarrow\left[(\forall x)\left(\left(M_{2} \mid z\right) x \Rightarrow P x\right) \wedge E v\right] .
\end{aligned}
$$

Also, the following formula is strongly valid $T^{I Q} \vdash\left(\left(M_{2} \mid z\right) x \Rightarrow P x\right) \Rightarrow\left(\left(\left(M_{2} \mid z^{\prime}\right) x \Rightarrow S x\right) \Rightarrow\right.$
$\left.\left(\left(\left(M_{2} \mid z\right) x \&\left(M_{2} \mid z^{\prime}\right) x\right) \Rightarrow(S x \wedge P x)\right)\right)$. By the generalization rule $(\forall x)$ and by properties of quantifiers we obtain $T^{I Q} \vdash(\forall x)\left(\left(M_{2} \mid z\right) x \Rightarrow P x\right) \Rightarrow\left((\forall x)\left(\left(M_{2} \mid z^{\prime}\right) x \Rightarrow\right.\right.$ $\left.S x) \Rightarrow\left((\exists x)\left(\left(M_{2} \mid z\right) x \&\left(M_{2} \mid z^{\prime}\right) x\right) \Rightarrow(\exists x)(S x \wedge P x)\right)\right)$. By Ł-FTT properties we obtain

$$
\begin{aligned}
& T^{I Q} \vdash\left[(\forall x)\left(\left(M_{2} \mid z\right) x \Rightarrow P x\right) \wedge E v\right] \Rightarrow \\
& \Rightarrow\left((\forall x)\left(\left(M_{2} \mid z^{\prime}\right) x \Rightarrow S x\right) \Rightarrow\right. \\
& \left.\Rightarrow\left((\exists x)\left(\left(M_{2} \mid z\right) x \&\left(M_{2} \mid z^{\prime}\right) x\right) \Rightarrow(\exists x)(S x \wedge P x)\right)\right) .
\end{aligned}
$$

By Ł-FTT properties we obtain

$$
\begin{aligned}
& T^{I Q} \vdash\left[(\forall x)\left(\left(M_{2} \mid z^{\prime}\right) x \Rightarrow S x\right) \wedge E v^{\prime}\right] \Rightarrow \\
& \Rightarrow\left(\left[(\forall x)\left(\left(M_{2} \mid z\right) x \Rightarrow P x\right) \wedge E v\right] \Rightarrow\right. \\
& \left.\Rightarrow\left((\exists x)\left(\left(M_{2} \mid z\right) x \&\left(M_{2} \mid z^{\prime}\right) x\right) \Rightarrow(\exists x)(S x \wedge P x)\right)\right) .
\end{aligned}
$$

By adjunction we obtain

$$
\begin{aligned}
& T^{I Q} \vdash\left[(\forall x)\left(\left(M_{2} \mid z\right) x \Rightarrow P x\right) \wedge E v\right] \Rightarrow \\
& \Rightarrow\left(\left[(\forall x)\left(\left(M_{2} \mid z^{\prime}\right) x \Rightarrow S x\right) \wedge E v^{\prime}\right] \Rightarrow\right. \\
& \left.\Rightarrow\left((\exists x)\left(\left(M_{2} \mid z\right) x \&\left(M_{2} \mid z^{\prime}\right) x\right) \Rightarrow(\exists x)(S x \wedge P x)\right)\right) .
\end{aligned}
$$

By applying transitivity on formulas (5),(6) we obtain

$$
\begin{aligned}
& T^{I Q} \vdash\left((\forall x)\left(M_{1} \Rightarrow P x\right) \&\right. \\
&\left.\&\left[(\forall x)\left(\left(M_{2} \mid z\right) x \Rightarrow M_{1} x\right) \wedge E v\right]\right) \Rightarrow \\
& \Rightarrow\left(\left[(\forall x)\left(\left(M_{2} \mid z^{\prime}\right) x \Rightarrow S x\right) \wedge E v^{\prime}\right] \Rightarrow\right. \\
&\left.\Rightarrow\left((\exists x)\left(\left(M_{2} \mid z\right) x \&\left(M_{2} \mid z^{\prime}\right) x\right) \Rightarrow(\exists x)(S x \wedge P x)\right)\right) .
\end{aligned}
$$

By adjunction we obtain

$$
\begin{aligned}
& T^{I Q} \vdash\left((\forall x)\left(M_{1} \Rightarrow P x\right) \&\right. \\
& \&\left[(\forall x)\left(\left(M_{2} \mid z\right) x \Rightarrow M_{1} x\right) \wedge E v\right] \& \\
& \&\left[(\forall x)\left(\left(M_{2} \mid z^{\prime}\right) x \Rightarrow S x\right) \wedge E v^{\prime}\right] \& \\
& \left.\left.\&(\exists x)\left(\left(M_{2} \mid z\right) x \&\left(M_{2} \mid z^{\prime}\right) x\right)\right) \Rightarrow(\exists x)(S x \wedge P x)\right) .
\end{aligned}
$$

By the generalization rule $(\forall z),\left(\forall z^{\prime}\right)$ and by properties of quantifiers we obtain

$$
\begin{aligned}
& T^{I Q} \vdash(\exists z)\left(\exists z^{\prime}\right)\left((\forall x)\left(M_{1} \Rightarrow P x\right) \&\right. \\
& \&\left[(\forall x)\left(\left(M_{2} \mid z\right) x \Rightarrow M_{1} x\right) \wedge E v\right] \& \\
& \&\left[(\forall x)\left(\left(M_{2} \mid z^{\prime}\right) x \Rightarrow S x\right) \wedge E v^{\prime}\right] \&(\exists x)\left(\left(M_{2} \mid z\right) x \&\right. \\
& \left.\left.\left.\&\left(M_{2} \mid z^{\prime}\right) x\right)\right) \Rightarrow(\exists x)(S x \wedge P x)\right) .
\end{aligned}
$$

Finally, we replace $E v:=\operatorname{BiEx}\left(\left(\mu M_{2}\right)\left(M_{2} \mid z\right)\right)$ and $E v^{\prime}:=\neg S m \overline{\mathbf{v}}\left(\left(\mu M_{2}\right)\left(M_{2} \mid z^{\prime}\right)\right)$ and we get the proof of the syllogism APKI. The proofs of the syllogisms ATTI and AKPI are similar. For the syllogism ATTI we replace $E v:=B i V e\left(\left(\mu M_{2}\right)\left(M_{2} \mid z\right)\right)$ and $E v^{\prime}:=B i V e\left(\left(\mu M_{2}\right)\left(M_{2} \mid z^{\prime}\right)\right)$. For the syllogism AKPI we replace $E v:=\neg \operatorname{Sm} \overline{\mathbf{v}}\left(\left(\mu M_{2}\right)\left(M_{2} \mid z\right)\right)$ and $E v^{\prime}:=$ $\operatorname{BiEx}\left(\left(\mu M_{2}\right)\left(M_{2} \mid z^{\prime}\right)\right)$. 
If we have proofs of these syllogisms we can use knowledge of their strong validity and monotonicity to prove other syllogisms.

Theorem 3. Let syllogisms AAII, APKI, ATTI, AKPI, AIAI be strongly valid in $T^{I Q}$. Then also the following syllogisms are strongly valid in $T^{I Q}$ :

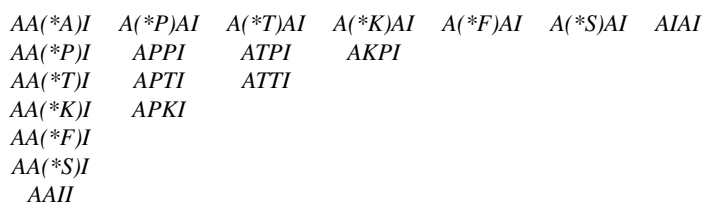

Proof. For the proofs of syllogisms in the first column, we use strong validity of syllogism AAII and apply Theorem 1 to it. The proofs of syllogisms in other columns are obtained analogously except for syllogisms A(*F)AI, $\mathbf{A}(* \mathbf{S}) \mathbf{A I}$. We get the proofs of these syllogisms by applying Theorem 1 on the strongly valid syllogism AIAI.

In Theorem 3 we ordered syllogisms according to the monotonicity and we created a triangle. We can see that vertexes of this triangle are classical syllogisms and all the intermediate syllogisms lie between them. We can also see that we get other syllogisms by strengthening one of the premises.

Syllogisms APPI, ATPI, AKPI, APTI, ATTI, APKI are non-trivial. Non-trivial syllogisms contain two intermediate quantifiers. Also in proof of non-trivial syllogism APKI we can see formula $(\exists x)\left(\left(M_{2} \mid z\right) x \&\left(M_{2} \mid z^{\prime}\right) x\right)$. This formula is called common presupposition [6].

Next, we present the proof of syllogism AA(*A)I-II.

Theorem 4. The syllogism $\mathbf{A A}(* \boldsymbol{A}) \boldsymbol{I}$ is strongly valid in $T^{I Q}$.

$$
\begin{array}{ll} 
& (\forall x)\left(M_{1} x \Rightarrow P x\right) \\
\text { Proof. AA(*A)I-II: } & (\forall x)\left(M_{2} x \Rightarrow M_{1} x\right) \\
& (\forall x)\left(M_{2} x \Rightarrow S x\right) \&(\exists x)\left(M_{2} x\right) \\
& (\exists x)(S x \wedge P x)
\end{array}
$$

The following formulas are strongly valid in $T^{I Q}$ : $T^{I Q} \vdash\left(\left(M_{1} x \Rightarrow P x\right) \&\left(M_{2} x \Rightarrow M_{1} x\right)\right) \Rightarrow\left(M_{2} x \Rightarrow P x\right)$ and $T^{I Q} \vdash\left(\left(M_{2} x \Rightarrow P x\right) \&\left(M_{2} x \Rightarrow S x\right)\right) \Rightarrow\left(M_{2} x \Rightarrow\right.$ $(S x \wedge P x))$. By applying adjunction on the second formula and by transitivity we obtain

$$
\begin{aligned}
& T^{I Q} \vdash\left(\left(M_{1} x \Rightarrow P x\right) \&\left(M_{2} x \Rightarrow M_{1} x\right)\right) \Rightarrow \\
& \quad \Rightarrow\left(\left(M_{2} x \Rightarrow S x\right) \Rightarrow\left(M_{2} x \Rightarrow(S x \wedge P x)\right)\right) .
\end{aligned}
$$

By adjunction we obtain

$$
\begin{aligned}
& T^{I Q} \vdash\left(M_{1} x \Rightarrow P x\right) \Rightarrow\left(\left(M_{2} x \Rightarrow M_{1} x\right) \Rightarrow\right. \\
& \left.\quad \Rightarrow\left(\left(M_{2} x \Rightarrow S x\right) \Rightarrow\left(M_{2} x \Rightarrow(S x \wedge P x)\right)\right)\right) .
\end{aligned}
$$

By the generalization rule $(\forall x)$ and by properties of quantifiers we obtain

$$
\begin{gathered}
T^{I Q} \vdash(\forall x)\left(M_{1} x \Rightarrow P x\right) \Rightarrow\left((\forall x)\left(M_{2} x \Rightarrow M_{1} x\right) \Rightarrow\right. \\
\left.\Rightarrow\left((\forall x)\left(M_{2} x \Rightarrow S x\right) \Rightarrow\left((\exists x) M_{2} x \Rightarrow(\exists x)(S x \wedge P x)\right)\right)\right)
\end{gathered}
$$

Finally, by adjunction we get

$$
\begin{aligned}
& T^{I Q} \vdash\left((\forall x)\left(M_{1} x \Rightarrow P x\right) \&(\forall x)\left(M_{2} x \Rightarrow M_{1} x\right) \&\right. \\
& \left.\quad \&(\forall x)\left(M_{2} x \Rightarrow S x\right) \&(\exists x) M_{2} x\right) \Rightarrow(\exists x)(S x \wedge P x) .
\end{aligned}
$$

We can see that the presupposition in this syllogism

\begin{tabular}{|c|c|c|c|c|c|c|}
\hline \multirow{2}{*}{$\begin{array}{l}\text { Theorem } \\
\text { are }\end{array}$} & \multirow{2}{*}{\multicolumn{2}{|c|}{ atrongly }} & \multirow{2}{*}{\multicolumn{2}{|c|}{$\begin{array}{l}\text { These } \\
\text { valid }\end{array}$}} & \multicolumn{2}{|c|}{ syllogisms } \\
\hline & & & & & in & $T^{I Q}$. \\
\hline$E A(* A) O$ & $E(* P) A O$ & $E(* T) A O$ & $E\left({ }^{*} K\right) A O$ & $E(* F) A O$ & $E(* S) A O$ & EIAO \\
\hline$E A(* P) O$ & ЕРPO & ЕТРО & $E K P O$ & & & \\
\hline$E A(* T) O$ & EPTO & ETTO & & & & \\
\hline$E A(* K) O$ & EPKO & & & & & \\
\hline$E A(* F) O$ & & & & & & \\
\hline$E A(* S) O$ & & & & & & \\
\hline EAIO & & & & & & \\
\hline
\end{tabular}
can be located on the second or the third premise. If we look at syllogisms $\mathbf{A}(* \mathbf{P}) \mathbf{A I}$ and $\mathbf{A A}(* \mathbf{P}) \mathbf{I}$ we can see that in these syllogisms one of the premises is weaker than in syllogism $\mathbf{A} \mathbf{A}\left(\mathbf{A}^{*}\right) \mathbf{I}$, but for these syllogisms, the position of the presupposition is already fixed and the position of the presupposition is on the premise with the intermediate quantifier.

In Figure-II following syllogisms are also strongly valid.

Proof. The procedure for proving this group of syllogisms is similar to the procedure we have shown in Theorem 2 and Theorem 3.

\subsubsection{Converting a Syllogism with Three Premises to Syllogisms with Two Premises}

The main objective of this subsection is to show that syllogisms with more premises can be transformed into generalized Peterson's syllogisms. The idea to use very well known Peterson's syllogisms follows from the syntactic construction of proofs of syllogisms based on transitivity.

We focus on the syllogisms of Figure-II. In general, these syllogisms look as follows: 


\section{Figure-II}

$$
\begin{gathered}
P_{1}: Q_{1} M_{1} \text { is } P \\
P_{2}: Q_{2} M_{2} \text { is } M_{1} \\
P_{3}: Q_{3} M_{2} \text { is } S \\
\hline C: Q_{4} S \text { is } P
\end{gathered}
$$

We can split this Figure into two syllogisms with two premises:

\section{Figure-I $\quad$ Figure-III}

$$
\begin{array}{cc}
P_{1}: Q_{1} M_{1} \text { is } P & C_{M}: Q_{M} M_{2} \text { is } P \\
P_{2}: Q_{2} M_{2} \text { is } M_{1} & P_{3}: Q_{3} M_{2} \text { is } S \\
\hline C_{M}: Q_{M} M_{2} \text { is } P & C: Q_{3} S \text { is } P
\end{array}
$$

or

$$
\begin{array}{ll}
\multicolumn{3}{c}{\text { The second case }} \\
\text { Figure-III } & \text { Figure-I } \\
P_{2}: Q_{2} M_{2} \text { is } M_{1} & P_{1}: Q_{1} M_{1} \text { is } P \\
P_{3}: Q_{3} M_{2} \text { is } S & C_{N}: Q_{N} S \text { is } M_{1} \\
\hline C_{N}: Q_{N} S \text { is } M_{1} & C: Q_{3} S \text { is } P
\end{array}
$$

In the first case from premises $P_{1}, P_{2}$ we infer the conclusion $C_{M}$. Then we use this conclusion $C_{M}$ as the premise in the other syllogism and from $C_{M}$ and $P_{3}$, we infer the final conclusion $C$.

In the second case from premises $P_{2}, P_{3}$ we infer the conclusion $C_{N}$. Then we use this conclusion $C_{N}$ as the second premise in the other syllogism and from premises $P_{1}, C_{N}$ we infer the final conclusion $C$.

We demonstrate this idea on the following example:

All bacterial illnesses are treated with antibiotics. Most seasonal illnesses are bacterial.

Most seasonal illnesses are flu-like.

Some flu-like illnesses are treated with antibiotics.

We can see that this syllogism is an example of the syllogism ATTI-II.

Example 1. We want to show the strong validity of the syllogism ATTI-II using the transformation into two separate Peterson's syllogisms.

\section{ATTI-II}

\begin{tabular}{ccc}
$P_{1}:$ & All & $M_{1}$ are $P$ \\
$P_{2}:$ & Most & $M_{2}$ are $M_{1}$ \\
$P_{3}:$ & Most & $M_{2}$ are $S$ \\
\hline$C:$ & Some & $S$ are $P$
\end{tabular}

We start with the first case.

ATT-I

$P_{1}:$ All $M_{1}$ are $P \quad C_{M}$ : Most $M_{2}$ are $P$

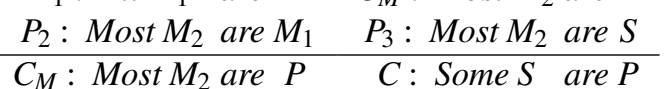

The syllogism ATT is strongly valid in Figure-I ([4]). We can see that from premise $P_{1}(A l l)$ and premise $P_{2}$ (Most) we inferred the conclusion $C_{M}$ (Most). Then we know that syllogism TTI is strongly valid in Figure-III. We can see that from $C_{M}$ (Most) and from $P_{3}$ (Most) we can infer the final conclusion C (Some).

\section{TTI-III}

AII-I

\begin{tabular}{lc}
$P_{2}:$ Most $M_{2}$ are $M_{1}$ & $P_{1}:$ All $M_{1}$ are $P$ \\
$P_{3}:$ Most $M_{2}$ are $S$ & $C_{N}:$ Some $S$ are $M_{1}$ \\
\hline$C_{N}:$ Some $S$ are $M_{1}$ & $C:$ Some $S$ are $P$
\end{tabular}

In the second case, we will first observe strongly valid syllogisms with two premises on Figure-III which contain the intermediate quantifier most in both premises. In [4] we can find that the syllogism TTI fulfills our condition. Then we from $P_{2}$ (Most) and $P_{3}$ (Most) infer conclusion $C_{N}$ (Some). This conclusion we use as the second premise, so we will search for syllogisms that contain in premises intermediate quantifiers all, some in Figure-I. We can see that syllogism AII fulfils our condition. Then from $P_{1}$ (all) and $C_{N}$ (Some) we infer the final conclusion C (Some).

\section{Evaluation of Syllogisms with Three Premises}

In this section, we will propose an algorithm of evaluation of intermediate quantifiers expressed by formula (3). We will then apply the said algorithm to evaluating generalized syllogisms with three premises.

\subsection{Evaluation of Intermediate Quantifiers}

In theory $T^{I Q}$, formula (3) can be interpreted as the following function:

$$
\begin{gathered}
\left(Q_{E v}^{\forall} x\right)(B, A)= \\
\mathscr{M}\{(\exists z)[(\forall x)((B \mid z) x \Rightarrow A x) \wedge E v((\mu B)(B \mid z))]\}= \\
=\bigvee_{z \subseteq B}\{\mathscr{M}[(\forall x)((B \mid z) x \Rightarrow A x)] \wedge \mathscr{M}[E v((\mu B)(B \mid z))]\}= \\
=\bigvee_{z \subseteq B}\left\{\bigwedge_{x \in M}[(B \mid z) x \rightarrow A x] \wedge[f(F(B)(B \mid z))]\right\},
\end{gathered}
$$

where $\rightarrow$ represents the Eukasiewicz implication, $f$ is a non-decreasing function representing a particular evaluative expression and $F$ stands for a measure of fuzzy sets.

\subsubsection{The Algorithm}

For given non-empty fuzzy sets $B, A$ and an evaluative linguistic expression $E v$, Algorithm 1 returns the membership degree of the intermediate quantifier. 


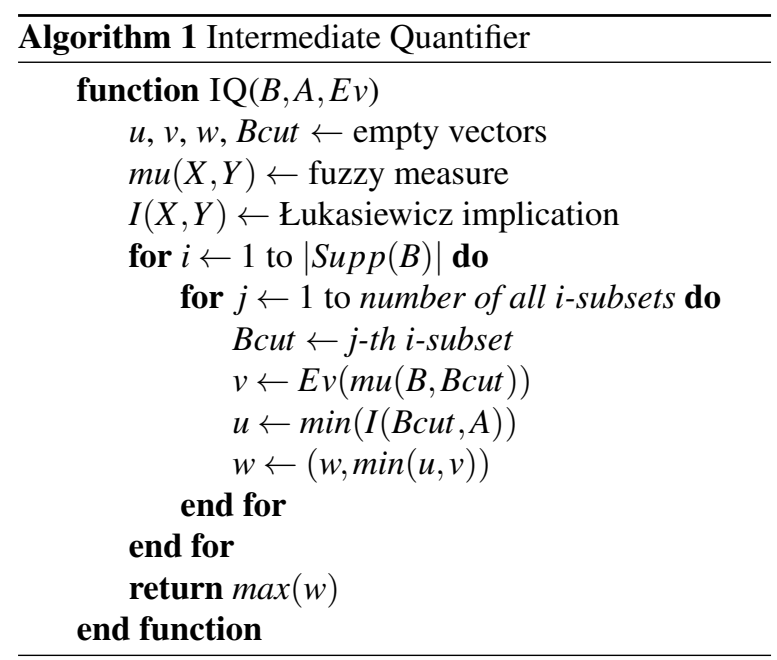

\subsubsection{Example of a Valid Syllogism}

Now we will propose an example of a generalized syllogism with three premises and we will show whether it is strongly valid in $T^{I Q}$ using the proposed algorithm implemented using the $\mathrm{R}$ programming language.

Let us consider the following syllogism.

All mild symptoms of COVID-19 are flu-like symptoms. Almost all cold symptoms are mild symptoms of COVID-19. Most cold symptoms are short-term symptoms.

Some short-term symptoms are flu-like symptoms..

This is the syllogism APTI-II consisting of the following formulas.

$$
\begin{aligned}
& (\forall x)\left(M_{1} x \Rightarrow P x\right) \\
& (\exists z)\left[(\forall x)\left(\left(M_{2} \mid z\right) x \Rightarrow M_{1} x\right) \wedge \operatorname{BiEx}\left(\left(\mu M_{2}\right)\left(M_{2} \mid z\right)\right)\right] \\
& \left(\exists z^{\prime}\right)\left[(\forall x)\left(\left(M_{2} \mid z^{\prime}\right) x \Rightarrow S x\right) \wedge \operatorname{BiVe}\left(\left(\mu M_{2}\right)\left(M_{2} \mid z^{\prime}\right)\right)\right] \\
& \hline(\exists x)(S x \wedge P x)
\end{aligned}
$$

Let $U$ be a set of symptoms. Let Mild be a formula representing "mild symptoms of COVID-19" which is interpreted by fuzzy set $M_{1}, \mathscr{M}($ Mild $)=M_{1} \subseteq U$. Let Cold be a formula representing "cold symptoms" interpreted by $\mathscr{M}($ Cold $)=M_{2} \subsetneq U$. Let "flu-like symptoms" be represented by formula $F l u$ interpreted by $\mathscr{M}(F l u)=P \subseteq U$. Finally, let formula Short represent "short-term symptoms" interpreted by $\mathscr{M}($ Short $)=$ $S \cong U$.

$M_{1}, M_{2}, P$ and $S$ are fuzzy sets given by the following table.

\begin{tabular}{|c||c|c|c|c|}
\hline & $M_{1}$ & $M_{2}$ & $P$ & $S$ \\
\hline \hline$u_{1}$ & 0.35 & 0.31 & 0.44 & 0.11 \\
$u_{2}$ & 0.77 & 0.14 & 0.39 & 0.14 \\
$u_{3}$ & 0.32 & 0.32 & 0.08 & 0.33 \\
$u_{4}$ & 0.94 & 0.74 & 0.53 & 0.71 \\
$u_{5}$ & 0.26 & 0.49 & 0.23 & 0.75 \\
$u_{6}$ & 0.59 & 0.63 & 0.28 & 0.72 \\
$u_{7}$ & 0.24 & 0.02 & 0.09 & 0.40 \\
$u_{8}$ & 0.57 & 0.77 & 0.61 & 0.21 \\
$u_{9}$ & 0.78 & 0.48 & 0.83 & 0.52 \\
$u_{10}$ & 0.59 & 0.31 & 0.10 & 0.36 \\
\hline
\end{tabular}

Table 1: Fuzzy sets $M_{1}, M_{2}, P, S \subsetneq U$

We used the algorithm and arrived at the following evaluations of formulas.

$\mathscr{M}\left(P_{1}\right)=0.91, \mathscr{M}\left(P_{2}\right)=0.77, \mathscr{M}\left(P_{3}\right)=0.44, \mathscr{M}(C)=0.53$

From Definition 4 we get the inequality $0.12 \leq 0.53$.

Therefore the syllogism is strongly valid in $T^{I Q}$.

\subsubsection{Example of an Invalid Syllogism}

Let us consider the following syllogism.

All old people are high risk for COVID-19.

Almost all obese people are high risk for COVID-19.

Many obese people are at risk of heart attacks.

All people at risk of heart attacks are old.

This is the syllogism APKA-III consisting of the following formulas.

$$
\begin{aligned}
& (\forall x)\left(P x \Rightarrow M_{1} x\right) \\
& (\exists z)\left[(\forall x)\left(\left(M_{2} \mid z\right) x \Rightarrow M_{1} x\right) \wedge \operatorname{BiEx}\left(\left(\mu M_{2}\right)\left(M_{2} \mid z\right)\right)\right] \\
& (\exists z)\left[(\forall x)\left(\left(M_{2} \mid z\right) x \Rightarrow S x\right) \wedge \neg \operatorname{Sm}\left(\left(\mu M_{2}\right)\left(M_{2} \mid z\right)\right)\right] \\
& (\forall x)(S x \Rightarrow P x)
\end{aligned}
$$

Let $V$ be a set of people. Let $O l d$ be a formula representing "old people" which is interpreted by fuzzy set $P, \mathscr{M}($ Old $)=P \subseteq V$. Let $C o v$ be a formula representing "people high risk for COVID-19" which is interpreted by $\mathscr{M}(\mathrm{Cov})=M_{1} \subsetneq V$. Let "obese people" be represented by formula Obese interpreted by $\mathscr{M}($ Obese $)=M_{2} \subseteq V$. Finally, let formula Heart represent "people at high risk of heart attacks" interpreted by $\mathscr{M}($ Heart $)=S \Subset V$.

$M_{1}, M_{2}, P$ and $S$ are fuzzy sets given by the following table. 


\begin{tabular}{|c||c|c|c|c|}
\hline & $M_{1}$ & $M_{2}$ & $P$ & $S$ \\
\hline \hline$v_{1}$ & 0.72 & 0.63 & 0.33 & 0.01 \\
\hline$v_{2}$ & 0.80 & 0.51 & 0.68 & 0.53 \\
\hline$v_{3}$ & 0.15 & 0.05 & 0.21 & 0.66 \\
\hline$v_{4}$ & 0.93 & 0.21 & 0.90 & 0.03 \\
\hline$v_{5}$ & 0.48 & 0.72 & 0.41 & 0.37 \\
\hline$v_{6}$ & 0.76 & 0.63 & 0.54 & 0.49 \\
\hline$v_{7}$ & 0.60 & 0.29 & 0.12 & 0.98 \\
\hline$v_{8}$ & 0.97 & 0.96 & 0.66 & 0.75 \\
\hline$v_{9}$ & 0.57 & 0.33 & 0.93 & 0.36 \\
\hline$v_{10}$ & 0.74 & 0.21 & 0.13 & 0.59 \\
\hline
\end{tabular}

Table 2: Fuzzy sets $M_{1}, M_{2}, P, S \Subset V$

Using the algorithm previously proposed we arrived at these evaluations of each formula.

$\mathscr{M}\left(P_{1}\right)=0.64, \mathscr{M}\left(P_{2}\right)=0.76, \mathscr{M}\left(P_{3}\right)=1, \mathscr{M}(C)=0.14$

From Definition 4 we get the inequality $0.4 \leq 0.14$ which does not hold, therefore the syllogism is not valid in $T^{I Q}$.

\section{Conclusion}

This paper extended basic Peterson's quantifiers by new forms and provided syntactic proofs of corresponding syllogisms with three premises. Using their strong validity and the monotonicity property, other syllogisms were proven. The following subsection demonstrated another approach to proving the strong validity of these syllogisms by transforming them into two syllogisms with two premises.

In the second part of the paper, an algorithm for the evaluation of intermediate quantifiers was proposed and subsequently used in verifying the validity of generalized syllogisms with three premises.

The idea for the future is to design an algorithm with which we will be able to verify the validity of syllogisms with $n$ premises using known Peterson's rules.

\section{Acknowledgement}

The work was supported from ERDF/ESF by the project "Centre for the development of Artificial Intelligence Methods for the Automotive Industry of the region" No. CZ.02.1.01/0.0/0.0/17-049/0008414.

\section{References}

[1] P. Andrews, An Introduction to Mathematical Logic and Type Theory: To Truth Through Proof, Kluwer, Dordrecht, 2002.

[2] D. Dubois, H. Prade, On fuzzy syllogisms, Comput.Intell. 4 (1988) 171-179.
[3] P. Murinová, Generalized intermediate syllogisms with more premises, in: Proc. FUZZ-IEEE 2019, New Orleands, USA, 2019.

[4] P. Murinová, V. Novák, A formal theory of generalized intermediate syllogisms, Fuzzy Sets and Systems 186 (2013) 47-80.

[5] P. Murinová, V. Novák, Analysis of generalized square of opposition with intermediate quantifiers, Fuzzy Sets and Systems 242 (2014) 89113.

[6] P. Murinová, V. Novák, The theory of intermediate quantifiers in fuzzy natural logic revisited and the model of "many", Fuzzy sets and systems 388 (2020) 56-89.

[7] V. Novák, On fuzzy type theory, Fuzzy Sets and Systems 149 (2005) 235-273.

[8] V. Novák, A comprehensive theory of trichotomous evaluative linguistic expressions, Fuzzy Sets and Systems 159 (22) (2008) 2939-2969.

[9] V. Novák, A formal theory of intermediate quantifiers, Fuzzy Sets and Systems 159 (10) (2008) 1229-1246.

[10] V. Novák, S. Lehmke, Logical structure of fuzzy IF-THEN rules, Fuzzy Sets and Systems 157 (2006) 2003-2029.

[11] V. Novák, P. Murinová, A formal model of the intermediate quantifiers "a few", "several" and "a little”, in: Proc. IFSA-NAFIPS 2019, Lafayette, USA, 2019.

[12] V. Novák, I. Perfilieva, A. Dvořák, Insight into Fuzzy Modeling, Wiley \& Sons, Hoboken, New Jersey, 2016.

[13] M. Pereira-Fariña, F. Díaz-Hermida, A. Bugarín, On the analysis of set-based fuzzy quantified reasoning using classical syllogistics, Fuzzy Sets and Systems 214 (2013) 83-94.

[14] M. Pereira-Fariña, J. C. Vidal, F. Díaz-Hermida, A. Bugarín, A fuzzy syllogistic reasoning schema for generalized quantifiers, Fuzzy Sets and Systems 234 (2014) 79-96.

[15] P. Peterson, Intermediate Quantifiers. Logic, linguistics, and Aristotelian semantics, Ashgate, Aldershot, 2000.

[16] L. A. Zadeh, Syllogistic reasoning in fuzzy logic and its applications to usuality and reasoning with dispositions, IEEE Trans. Syst. Man Cybern. 15 (1985) 754-765. 